\title{
En torno al debate sobre la definicion del periodismo ambiental
}

\author{
Dr. Rogelio Fernández Reyes \\ Grupo de Investigación en Estructura, Historia y Contenidos de la Comunicación \\ Universidad de Sevilla
}

\section{RESUMEN}

La especialización periodística es un fenómeno del periodismo actual que va tomando cuerpo. Como muestra de ello, las diferentes facultades de Ciencias de la Información han ido incorporando en sus planes de estudio asignaturas denominadas, en un principio, Periodismo Especializado, y después, Información Periodística Especializada.

En cuanto a la conceptualización, la denominación de periodismo ambiental se ha consensuado entre los profesionales de la información. Si bien es verdad que tanto en el ámbito académico y en los cursos especializados aún no hay una designación homogénea, parece que acabará prevaleciendo el vocablo ambiental sobre ecológico, medioambiental o verde.

A la hora de definir periodismo ambiental hay que tener en cuenta las connotaciones que palabras como naturaleza, entorno, ecología están adquiriendo. Para nosotros se trata del ejercicio o periodismo especializado que atiende la información generada por la interacción del hombre o de los seres vivos con su entorno, o del entorno en sí.

\section{ABSTRAC}

The specialized journalism is a present journalism's phenomenon which is going to take shape. So, the different faculties of "Sciences of the Information" have gone incorporating in their syllabus subjects called, at first, Specialized Journalism, and later, Specilized Journalistic Information.

With regard to the conceptualization, the environmental journalism designation has been agreed between the information's professionals. Despite the fact that both in the academic ambit and in the specialized courses there isn't still a homogeneous designation, it looks as though it's going to finish prevailing the environmental term about ecological, environmental or green.

At teh moment of defining the environmental journalism, there is to take into account the connotations that words as nature, scene, ecology are acquiring. For us, it's about the specialized journalism that pay attention to the information produced by the man's interaction or the living creature's interaction with their environment or the scene interaction in itself.

Palabras claves: Periodismo ambiental/Especialidad periodística/Periodismo especializado.

Key words: Environmental Journalism/Journalism's Speciality/Specialized Journalism. 


\section{1.- Especialidad Periodística}

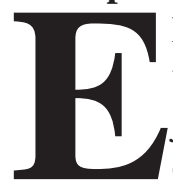

1 desarrollo tecnológico y científico del pasado siglo XX ha generado una explosión en el volumen de conocimientos. Ello ha quedado reflejado en el aumento de ediciones, en distintos soportes, de información especializada. Para el profesor Esteve Ramírez, la especialización periodística es, junto con las innovaciones tecnológicas, uno de los fenómenos más significativos del periodismo actual. Por una parte, los usuarios demandan una mayor profundización en las causas y consecuencias que se pueden derivar de los acontecimientos; por otra, los medios son conscientes de estas necesidades y de las posibilidades que ofrecen las tecnologías de la información (Esteve, 1997: 5).

Se ha pasado, pues, de la cultura abanico a la cultura mosaico (Esteve y Fernández, 1998: 7 y 8):

«Este incremento constante del saber y la ciencia comporta la necesidad de fragmentar la unidad del conocimiento humano a fin de permitir la mayor difusión del mismo (...) La parcelación del saber puede ser beneficiosa para las ciencias particulares en virtud de su mayor capacidad de profundización, pero genera el creciente riesgo de la incomunicación entre las distintas ramas del saber".

Para salvar las disfunciones de la relación entre la ciencia y la sociedad, Fernández del Moral aboga por la especialización periodística como nexo entre ambos (Fernández del Moral, 1983: 7).

En su libro Periodismo Especializado, Montserrat Quesada propone como definición de dicha materia:

«el que resulta de la aplicación minuciosa de la metodología periodística de investigación a los múltiples ámbitos temáticos que conforman la realidad social, condicionada siempre por el medio de comunicación que se utilice como canal, para dar respuesta a los intereses y necesidades de las nuevas audiencias sectoriales" (Quesada, 1998: 23).

En 1974, los profesores Pedro Orive y Concha Fagoaga ya consideraban el periodismo especializado como

«un nuevo sistema de vertebración de la información que se apoya sobre el experto que trabaja un área concreta y determinada dentro de un medio también concreto y determinado con las características de profundización y fiabilidad en los mensajes que transmite" (Orive y Fagoaga en Fernández del Moral, 1983: 13).

En aquellos años, recuerda el profesor Orive, nació, como disciplina de segundo ciclo, Periodismo Especializado. Esta asignatura, que luego pasó a deno- 
minarse Información Periodística Especializada, se impartía en el quinto curso de la sección de Periodismo, a continuación de Estructura de la Información Periodística.

Es oportuno distinguir los conceptos Periodismo Ambiental y Periodismo Especializado para evitar confusiones. Según el catedrático Caminos Marcet:

«Si utilizamos una designación altamente precisa diremos que el Periodismo Ambiental forma parte del Periodismo Especializado. Pero si decimos que el Periodismo Ambiental es una especialización periodística lo definimos con menor rigor y mayor imprecisión, porque todas las especializaciones periodísticas no tienen por qué formar parte del Periodismo Especializado"1.

En la Facultad de Ciencias de la Información de la Universidad de Sevilla se imparte una asignatura matriz denominada Información Periodística Especializada. De esta se derivan otras, de carácter optativo, como son Periodismo Cultural, Periodismo Deportivo, Periodismo Social, Periodismo Político y varias más. Mas ninguna de ellas se dedica a la materia ambiental.

En los borradores del nuevo Plan de Estudios de dicha Facultad se llegó a contemplar la disciplina Periodismo Científico y Ambiental, pero finalmente se omitió Ambiental por entenderse que estaba incorporado en Científico.

Como mostraremos más adelante, el periodismo ambiental como especialidad ha sido escasamente estudiado. En 1987, Montserrat Quesada apuntaba que el periodismo de investigación en esta materia era prácticamente inédito (Quesada, 1987: 122) y recogía como excepción la investigación del periodista Rafael Cid sobre el Parque Nacional de Doñana, publicado en agosto de 1982 en Cambio 16. Sin embargo, como atestigua Joaquín Fernández en Dos Siglos de Periodismo Ambiental, esta especialidad tiene una dilatada trayectoria.

En cuanto a los contenidos que abarca la especialidad periodística ambiental, el citado periodista Joaquín Fernández elaboró una lista que ha de "afrontar el informador ambiental como aproximación orientativa":

-Residuos (urbanos, industriales, agrícolas, hospitalarios, nucleares, suelos contaminados).

-Agua (ríos, pantanos, trasvases, regadíos, contaminación de aguas superficiales y subterráneas, depuración, nuevas tecnologías, plantas desalinizadoras, el canon de vertido, las confederaciones hidrográficas).

1 Dicha aclaración la propuso como miembro del Tribunal de la tesis titulada Periodismo Ambiental y Ecologismo: Tratamiento Informativo del Vertido de Aznalcóllar en El País, Edición de Andalucía (19981999) 
-Bosques (incendios, repoblaciones).

-Conservacionismo o protección de la naturaleza (espacios protegidos, parques naturales y nacionales, especies de fauna y flora en peligro de extinción, comercio y tráfico de animales, caza y pesca, biodiversidad, aves).

-Mares (contaminación marina, efectos del mar en el clima, recursos marinos, disminución de recursos pesqueros, especies en peligro de extinción (las ballenas como especie emblemática), la contaminación radiactiva de los mares, (los vertidos de residuos nucleares y otros residuos especialmente impactantes, mareas negras).

-Costas (especulación urbanística, regeneración de playas).

-Suelo (especulación urbanística, erosión y desertificación).

-Energía (energías convencionales, energías alternativas, la energía nuclear, los residuos nucleares, el ahorro y la eficiencia energética).

-Transporte (impactos ambientales de los diferentes medios de transporte, tráfico urbano, automóvil, automóviles del futuro).

-Atmósfera (contaminación atmosférica en general, capa de ozono, efecto invernadero, cambio climático).

-Problemas urbanos (contaminación, tráfico, ruido, zonas verdes, degradación del patrimonio arquitectónico).

-Agricultura (agricultura y ganadería intensivas y extensivas, agricultura ecológica, pesticidas, desarrollo rural, vías pecuarias).

-Nuevas tecnologías. Reconversión ecológica de los procesos industriales. Seguimiento de los numerosos programas de investigación relacionados con la problemática ambiental.

-La complejidad de la Administración ambiental en España (Unión Europea, fondos europeos, Agencia Europea de Medio Ambiente, organismos internacionales, convenios internacionales, Banco Mundial, Gatt, comercio internacional).

-Las organizaciones ecologistas. 
-Desarrollo sostenible y otros conceptos básicos del pensamiento ecológico.

-Turismo (impacto en el medio ambiente y en el uso de los recursos, turismo ecológico).

-Seguimiento de los estudios de impacto ambiental.

-Problemas de población.(Fernández, 1995: 18 y 19)

Otra relación es la que propuso la OCDE en un informe de 1992. En ella agrupó en cuatro los grandes problemas medioambientales de los países industrializados:

«Problemas atmosféricos: contaminaciones industriales, agrícolas, urbanas, lluvia ácida, capa de ozono, efecto invernadero, cambio climático, deforestación, energías renovables, biodiversidad, espacios protegidos, etc.

Problemas del agua: vertidos contaminantes, calidad del agua potable, contaminación de las aguas, acidificación de los lagos, planes hidrológicos, depuración de aguas, plantas desalinizadoras, aguas subterráneas, especies marinas en peligro de extinción, mareas negras, ecosistemas marinos

Problemas de residuos: recogida e incineración de residuos, transporte de residuos, residuos tóxicos y peligrosos, residuos urbanos y agrícolas, reciclaje, limpieza de vertederos, agricultura ecológica, etc.

Problemas de ruidos: contaminación acústica, etc."(Esteve y Fernández, 1998: 299)

\section{2.- Conceptualización}

La designación de periodismo ambiental se ha consensuado en los profesionales de la información. Otras alternativas presentadas fueron periodismo ecológico, medioambiental o verde.

Sobre el porqué prevaleció el término ambiental sobre su más cercano competidor, el vocablo ecológico, el periodista Joaquín Fernández apunta que se ha tratado de una preferencia de los sectores técnicos, profesionales y organismos administrativos:

«Lo ecológico, en cambio, resulta más conflictivo pues se asocia a ideología, compromiso, adoctrinamiento, dogma, militancia, lucha, etc. Es sin duda, un término cargado de intencionalidad y hasta de resonancias revolucionarias para muchos" (Fernández, 1995: 12). 
Benigno Varillas recoge en su trabajo Los movimientos ecologistas (Varillas, 1985: 23) que en los años de la transición se llegó a celebrar una pequeña reunión de periodistas dedicados en sus respectivas redacciones de prensa diaria y radio a la información ecologista, con el objetivo de constituir el colectivo Periodistas Ecologistas, pero la idea no llegó a prosperar nunca..

A finales de 1994 ya apareció el vocablo fijado en la Asociación de Periodistas de Información Ambiental (APIA). Con ello, los profesionales de la especialidad se desvinculaban de las confusiones que podía acarrear el nombre "ecológico" por su carga ideológica y se reafirmaban en lo ambiental como un ejercicio profesional.

Otras instituciones que tienen acuñados los términos de información ambiental son SinambA y CEIA. SinambA es un Sistema de Información Ambiental de Andalucía ubicado en la Isla de la Cartuja sevillana y dependiente de la Consejería de Medio Ambiente de la Junta de Andalucía. CEIA es un Centro de Estudios de Información Ambiental del Instituto Catalán de Tecnología.

Por otro lado, también es cierto que en los numerosos cursos, masters y premios sobre periodismo ambiental tienen otros títulos que no acaban de acuñarse de una manera homogénea: Curso de Especialización en Información Ecológica y Medioambiental, organizada por la Universidad Politécnica de Madrid en 1995; Diploma Universitario de Postgrado en Comunicación Medioambiental, del máster en Comunicación Científica programado por la Universidad Pompeu Fabra de Barcelona en el curso 96-97; Jornadas sobre Periodisme i medi ambient organizadas por la Unió de Periodistes en 1997; Curso Naturaleza y Medio Ambiente en Televisión, impartido en la Facultad de Comunicación de la Universidad de Navarra en 1998 (Montaño, 1999: 69 y 70); I Seminario sobre Periodismo y Medio Ambiente celebrado en Palma del Río en 1998; Curso de Verano Comunicación y Medio Ambiente organizado por la Universidad de Córdoba en julio de 2000 que no se llegó a celebrarse; o el II Certamen de "Joven Periodismo Ambiental", convocado en febrero de 2001.

En el ámbito académico aún no hay una definición unánime: los investigadores Esteve y Fernández del Moral designan el área como periodismo medioambiental (Esteve y Fernández, 1998: 298), mientras que en la reciente tesis de Miguel Montaño aparece como periodismo ambiental (Montaño, 1999).

Según Alberto Gómez y Antonio Machín, hay confusión en los términos medioambientales debido a que se dan significados diferentes a vocablos idénticos "según la profesión o nacionalidad de quienes los usan". El medio ambiente es un ámbito de estudio multidisciplinar por lo que "llegar a conocer y emplear bien todo ese caudal léxico es tarea harto difícil". (Gómez y Machín, 1997: 91). Un ejemplo de los diferentes significados queda reflejado en la definición que el profesor Angel Benito realiza de los términos ecología de la comunicación: 
«la disciplina que estudia los medios de comunicación, su distribución espacial y su organización, con relación al medio ambiente político, económico, cultural y social $^{2}$, con el fin de descubrir los desequilibrios comunicativos y proponer los correctivos pertinentes" (Benito, 1991: 401).

Sobre los términos ecología, naturaleza y medio ambiente encontramos en el Espasa-Calpe que el vocablo "ecología" expone: "se denomina así, modernamente, la parte de la biología que estudia el modo de vivir de los animales y las plantas y sus relaciones con los seres que los rodean" (VVAA, 1989: 2808). Realmente es un término moderno ya que el primero que lo utilizó fue el biólogo alemán Haeckel en 1868, unificando dos palabras griegas en una que significa ciencia del hábitat (Dreux, 1986: 3).

María Moliner lo define como "estudio de los organismos en relación con el medio"(Moliner, 1988: 1051). La Real Academia de la Lengua varía la definición en dos ediciones. En la vigésima propone "ciencia que estudia las relaciones existentes entre los seres vivientes y el medio ambiente en que viven" (VVAA, 1984: 522); en la vigésimo primera lo ajusta a "ciencia que estudia las relaciones de los seres vivos entre sí y con su entorno" (VVAA, 1992: 555).

Sobre el concepto naturaleza hay consenso entre La Real Academia (VVAA, 1992: 1012) y el Espasa (VVAA, 1989: 1203): “conjunto, orden y disposición de todas las entidades que componen el universo".

La composición medio ambiente es una expresión acuñada en 1800 por el danés Jens Baggesen, e introducida en el discurso biológico por Jakob von Uexkü11 (Boff, 1996: 15). El Espasa lo define de manera imprecisa dejando fuera el sector de la flora: "elemento en que vive o se mueve una persona, animal o cosa"(VVAA, 1989: 148). La Real Academia de la Lengua lo define como "conjunto de circunstancias físicas que rodean a los seres vivos"(VVAA, 1992: 953). Como novedad, en la vigésima primera edición dicha institución reconoce un vocablo nuevo: Medioambiental.

Podemos concluir, pues, que los vocablos ecología, medio ambiente y medioambiental, son palabras recientes en su uso generalizado. Y naturaleza está adquiriendo una acepción referida a los animales, plantas y medio natural.

El periodista ambiental Joaquín Fernández aclara cómo son utilizados dichos conceptos en el ejercicio de la profesión:

"Prescindiendo de toda ortodoxia conceptual, en los medios de comunicación utilizamos la palabra naturaleza para referirnos casi en exclusiva a la fauna y a la flora, y medio ambiente cuando se trata de cuestiones más relacionadas con el entorno urbano y los problemas derivados del desarrollo industrial y tecnológico.

2 El subrayado es nuestro. 
Ecología es un término global que usamos indistintamente para referirnos al ámbito científico, a los problemas de contaminación, al pensamiento ecológico o a los movimientos sociales que luchan contra la degradación del planeta" (Fernández, 1995: 12).

Los profesores Esteve y Del Moral apuntan como definición de esta área de especialización:

«la sección periodística que se ocupa de todas aquellas noticias relacionadas con la Naturaleza y su defensa"(Esteve y Fernández, 1998: 296).

Nosotros, a la hora de definir periodismo ambiental, preferimos proponer que se trata del ejercicio o periodismo especializado que atiende la información generada por la interacción del hombre o de los seres vivos con su entorno, o del entorno en sí.

De esta manera, se recogería la información que parte de la interacción del hombre/mujer con los espacios artificiales que ha creado, dado que la palabra Naturaleza está adquiriendo la connotación de medio natural.

Por últimpodemos puntualizar que la acepción entorno se circunscribe desde el espacio más inmediato hasta el planeta Tierra. El espacio interplanetario no se incluiría.

\section{Bibliografía utilizada:}

- BENITO, Angel (1991): Diccionario de ciencias y técnicas de la comunicación. Ediciones Paulinas, Madrid.

- BOFF Leonardo (1996): Ecología: Grito de la Tierra, Grito de los Pobres. Trotta, Madrid.

- DREUX, Philippe (1986): Introducción a la ecología. Alianza Editorial, Madrid.

- ESTEVE, Francisco (1997): Estudios sobre información periodística especializada. Fundación Universitaria San Pablo C.E.U., Valencia.

- ESTEVE y FERNÁNDEZ DEL MORAL (1998): Áreas de especialización periodística. Fragua, Madrid.

- FERNÁNDEZ DEL MORAL, Javier (1983): Modelos de comunicación científica para una información periodística especializada. Dossat, Madrid.

- FERNÁNDEZ DEL MORAL, Javier (1989): "La formación periodística especializada". En Información de medios, n¹, Madrid.

- FERNÁNDEZ, Rogelio (2001): Tesis doctoral Periodismo Ambiental y Ecologismo: Tratamiento informativo del vertido de Aznalcóllar en El País, Edición de Andalucía. Departamento de Periodismo de la Facultad de Ciencias de la Información de la Universidad de Sevilla. 
- FERNÁNDEZ SÁNCHEZ, Joaquín (1995): Periodismo Ambiental en España. Ministerio de Obras Públicas, Transportes y Medio Ambiente, Madrid.

- FERNÁNDEZ SÁNCHEZ, Joaquín (2001):Dos siglos de información ambiental. Caja de Ahorros del Mediterráneo.

- GÓMEZ, Alberto y MACHÍN, Antonio (1997): "El lenguaje del periodismo ambiental", II Congreso Nacional de Periodismo Ambiental. Apia, Madrid.

- MOLINER, María (1988): Diccionario del uso del español. Gredos, Madrid.

- MONTAÑO, Miguel (1999): La información ambiental en Andalucía: El programa <<Tierra y Mar>> de Canal Sur Andalucía. Tesis doctoral de la Facultad de Ciencias de la Información de la Universidad de Sevilla, Sevilla. Director: Ramón Reig. Inédita.

- ORIVE, Pedro y FAGOAGA, Concha (1974): La especialización en el periodismo. Dossat, Madrid.

- QUESADA, Montserrat (1987): La investigación periodística: el caso español. Ariel Comunicación, Barcelona.

- QUESADA, Montserrat (1998): Periodismo especializado. Ediciones Internacionales Universitarias, Madrid.

- VARILLAS, Benigno (1985): "Los movimientos ecologistas", en Cuadernos historia 16, $\mathrm{n}^{\circ}$ 131. Información y Revistas, Madrid.

- VVAA (1989): Espasa Calpe. Tomos 18, 34 y 37. Madrid

- VVAA (1984): Diccionario de la Lengua Española. Vigésima edición. EspasaCalpe, Madrid.

- VVAA (1992): Diccionario de la Lengua Española. Vigésima primera edición. Espasa-Calpe, Madrid 